\title{
New Insights in (Inter)Cellular Mechanisms by Heart Failure with Preserved Ejection Fraction
}

\author{
Carsten Tschöpe • Sophie Van Linthout
}

Published online: 5 September 2014

(C) The Author(s) 2014. This article is published with open access at Springerlink.com

\begin{abstract}
Recently, a new paradigm for the development of heart failure with preserved ejection fraction (HFpEF) has been proposed, which identifies a systemic proinflammatory state induced by comorbidities as the origin of microvascular endothelial cell inflammation and subsequent concentric cardiac remodeling and dysfunction. This review further discusses the pivotal role of the inflamed endothelium in the pathogenesis of HFpEF-specific cardiac remodeling. The potential importance of reciprocal interactions of the endothelium with cardiac fibroblasts and cardiomyocytes and with the cardiac neurohumoral response in this cardiac remodeling process is outlined.
\end{abstract}

Keywords Endothelial dysfunction - Comorbidities . HFpEF - Cardiac endothelium - Nitric oxide - TGF- $\beta$. Endothelial-to-mesenchymal transition . NOX . Inflammation - Cardiac fibrosis - Myofibroblast . Cardiomyocyte hypertrophy - Cardiomyocyte stiffness . Titin - Protein kinase A . Protein kinase G - cGMP . Sildenafil · Neurohumoral activation · Natriuretic peptides - BNP paradox - Neprilysin inhibitor . Mesenchymal stromal cells - Immunomodulation . Bidirectional cell interactions

\section{Tschöpe $(\bowtie)$}

Department of Cardiology and Pneumology, Charité, University Medicine Berlin, Campus Benjamin Franklin, Hindenburgdamm 30, 12200 Berlin, Germany

e-mail: carsten.tschoepe@charite.de

C. Tschöpe $\cdot$ S. Van Linthout

Berlin-Brandenburg Center for Regenerative Therapies, Charité, University Medicine Berlin, Campus Virchow Clinic, Berlin, Germany

S. Van Linthout

e-mail: sophie.van-linthout@charite.de

\author{
Abbreviations \\ Ang II Angiotensin II \\ ANP Atrial natriuretic peptide \\ BNP B-type natriuretic peptide \\ cAMP Cyclic adenosine monophosphate \\ cGMP Cyclic guanosine monophosphate \\ EndMT Endothelial-to-mesenchymal transition \\ $\mathrm{HFpEF}$ Heart failure with preserved ejection fraction \\ LV Left ventricular \\ MMP Matrix metalloproteinase \\ MSC Mesenchymal stromal cell \\ NADPH Nicotinamide adenine dinucleotide phosphate \\ NOX NADPH oxidase \\ PDE 2 Phosphodiesterase 2 \\ TNF Tumor necrosis factor \\ TGF Transforming growth factor \\ VCAM Vascular cell adhesion molecule
}

\section{Introduction}

Epidemiological studies demonstrate that $50 \%$ of all heart failure patients suffer from heart failure with preserved ejection fraction (HFpEF), which - in contrast to heart failure with reduced ejection fraction (HFREF) - cannot be adequately treated with current available therapeutical strategies [1]. Diabetes mellitus, obesity, hypertension, and COPD are the main comorbidities associated with HFpEF. [2] The high prevalence of $\mathrm{HFpEF}$ on the one hand and the rising prevalence of diabetes mellitus [3] and obesity [4] on the other hand, indicate the need for HFpEF-specific therapies. Further understanding of the underlying pathogenesis of $\mathrm{HFpEF}$ is required in view of finding novel treatment options. Diastolic stiffness underlying $\mathrm{HFpEF}$ is attributed to excessive 
myocardial collagen deposition and cardiomyocyte stiffness [5], of which the latter newly has been shown to be sufficient to induce HFpEF without any involvement of the extracellular matrix [6•]. Recently, a novel paradigm was postulated which identifies a systemic pro-inflammatory state induced by comorbidities as the origin of microvascular endothelial cell inflammation, which triggers HFpEF-specific, i.e., concentric, cardiac remodeling, and dysfunction $[7 \bullet \bullet]$.

This review gives a brief overview on how systemic inflammation induced by comorbidities influences endothelial cell behavior and signaling and how this affects the interaction of the endothelium with cardiomyocytes and cardiac fibroblasts, and the cardiac neurohumoral response, and subsequent cardiac remodeling. On the other hand, this review points out that those cells in turn influence the inflammation or directly the endothelium, aggravating the initial inflammatory process (Fig. 1). The impact of changes in substrate and subsequent energy metabolism on the pathogenesis of $\mathrm{HFpEF}$ is beyond the scope of this review.

\section{Endothelial Dysfunction and Heart Failure with Preserved Ejection Fraction}

The endothelium, the physical barrier between the blood and vascular wall, plays a pivotal role in cardiovascular homeostasis by regulating vasomotor tone, vascular permeability, and cardiac function [8]. The release of nitric oxide (NO) is crucial for the normal function of the endothelium: NO mediates vasodilatation, inhibits platelet aggregation, and protects the integrity of the endothelial layer via its anti-inflammatory

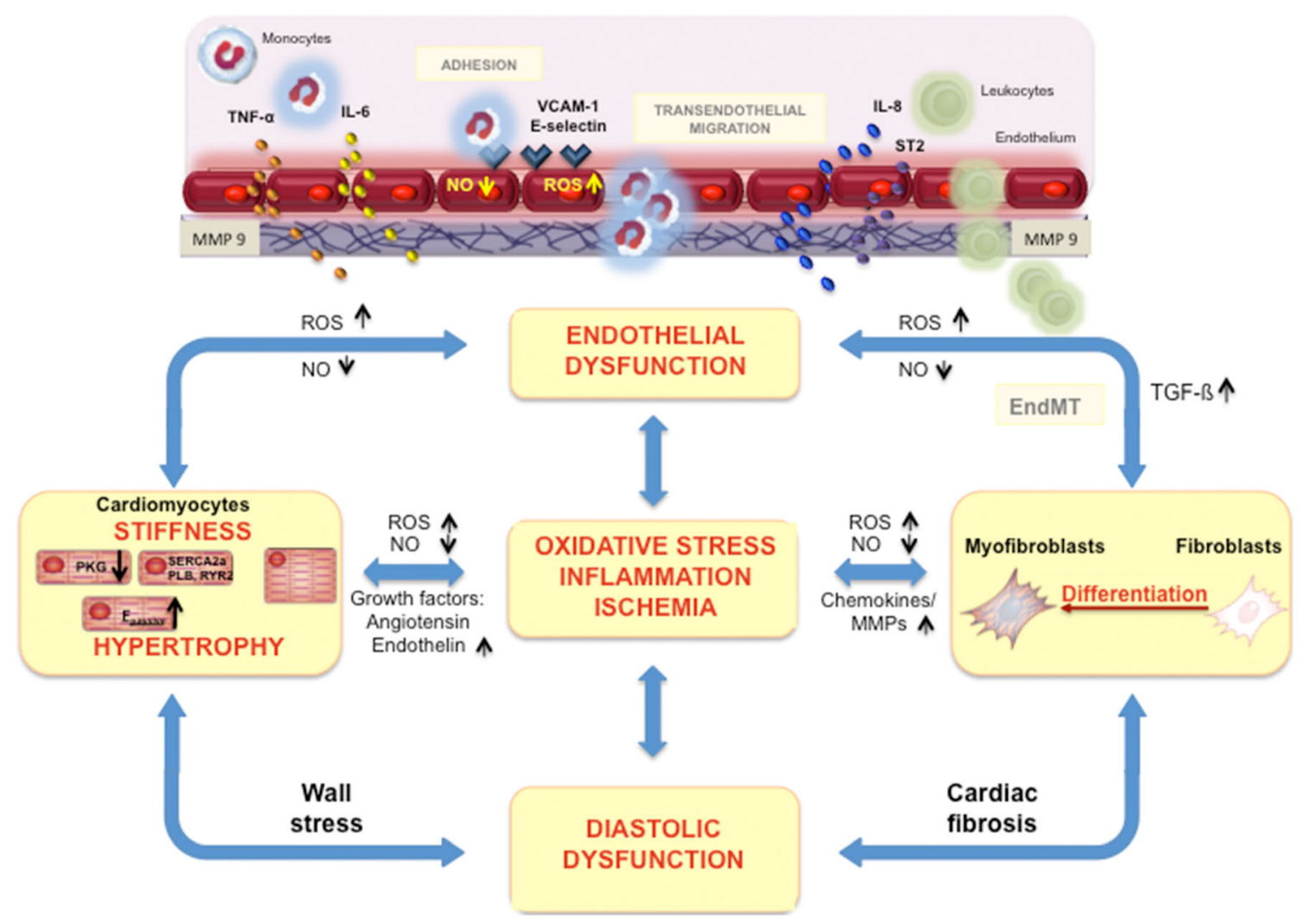

Fig. 1 Impact of endothelial dysfunction on the pathogenesis of HFpEF. Comorbodities such as obesity, hypertension, diabetes mellitus, microalbuminuria, iron deficiency, and hypercoagulability induce a systemic inflammatory state leading to increased VCAM-1 expression, subsequent monocyte adhesion, and infiltration of inflammatory cells releasing pro-inflammatory factors including interleukin (IL)-6, IL-8, tumor necrosis factor (TNF)- $\alpha$, and soluble ST2 (sST2), which promote endothelial dysfunction. The resulting endothelial dysfunction is associated with impaired NO bioavailibilty and ROS production, leading to the formation of peroxynitrite $\left(\mathrm{ONOO}^{-}\right)$, all of which induce lowering of protein kinase $\mathrm{G}$ (PKG) activity and subsequently titin hypophosphorylation and an increased resting tension $\left(F_{\text {passive }}\right)$ of cardiomyocytes. Intracellular calcium dysregulation involving dysfunction of the sarcoplasmic reticular adenosine triphosphate (ATP)-driven pump (SERCA), phospholamban (PLB), and/or ryanodine receptors (RYR) 2 further participates to cardiomyocyte stiffness. Low PKG activity and, among others, the release of endothelin- 1 by activated endothelial cells, contribute to cardiomyocyte hypertrophy. Furthermore, NO deprivation results in endothelial-to-mesenchymal transition (EndMT), a process by which endothelial cells transdifferentiate into (myo)fibroblasts. VCAM-1 and E-selectin expression on endothelial cells favors the adhesion of leukocytes, which by releasing transforming growth factor $\beta$ (TGF- $\beta$ ) stimulate EndMT and the conversion of fibroblasts to myofibroblasts and hereby cardiac fibrosis. By the release of, among others, chemokines (monocyte chemotractant protein-1) and the induction of matrix metalloproteinases, (myo)fibroblasts stimulate the inflammatory process. Cardiac fibrosis, cardiomyocyte hypertrophy, and/or cardiomyocyte stiffness contribute to wall stress and diastolic dysfunction 
[9-12], anti-apoptotic [13], and pro-angiogenic [14] properties.

The HFpEF-associated comorbidities lead to endothelial dysfunction, a condition characterized by impaired endothelium-dependent vasodilatation and "endothelial activation," referring to a state in which the endothelium loses its physiological properties and shifts towards a pro-inflammatory, pro-coagulatory, and vasoconstrictor state [15]. Particularly hyperglycemia, activation of the renin-angiotensin system, and tumor necrosis factor alpha (TNF- $\alpha$ ) underlie decreased NO bioavailability, the main characteristic of endothelial dysfunction, involving (1) deprivation of the substrate L-arginine via impaired recycling of L-citrulline [16]; (2) increased expression/activity of the natural competitor of endothelial NO synthase (eNOS), arginase [17]; (3) reduced expression of eNOS $[12,18]$; (4) a decreased ratio of the eNOS dimer to monomer [19]; (5) deprivation of the co-factor tetrabiopterin [20]; and/or (6) eNOS uncoupling, a phenomena by which eNOS produces superoxide $\left(\mathrm{O}_{2} \bullet\right)$ rather than $\mathrm{NO}[19,21]$. Hyperglycemia- or dyslipidemia-induced nicotinamide adenine dinucleotide phosphate (NAD $(\mathrm{P}) \mathrm{H})$ oxidase (NOX) [22] leads to $\mathrm{O}_{2} \bullet$ production and subsequent formation of peroxynitrite (ONOO-), which enhances eNOS uncoupling via reducing the affinity of eNOS for tetrabiopterin and via limiting the de novo synthesis of tetrabiopterin [23].

The cardiac endothelium comprises the endothelial cells of the coronary microvasculature, of the endocardium, and of the intramyocardial capillaries. Cardiomyocytes lie within the coronary microvascular network maximum $3 \mu \mathrm{m}$ from endothelial cells. This specific anatomical arrangement allows not only adequate blood supply but also facilitates the bidirectional communication among those cells [24-26]. The relevance of the cardiac endothelium in cardiac function follows from pioneer work from Brutsaert et al. [27] who demonstrated in vitro that the cardiac endocardial surface modulates the performance of cardiac muscle. Via coronary infusion of substance $\mathrm{P}$ leading to acute modulation of left ventricular (LV) function, Paulus et al. [28] provided later evidence that the cardiac endothelium is important for cardiac contractile function in humans potentially via the release of paracrine factors including NO, endothelin-1, natriuretic peptides, cytokines, and others. Both studies accentuate the significance of the cardiac endothelium for (acute) cardiomyocyte function. How the cardiac endothelium and consequently endothelial dysfunction or deterioration affects cardiomyocytes as well as cardiac fibroblasts and cardiac neurohumoral activation will further be addressed in the following paragraphs. The impact of the cardiac endothelium on those different cardiac cells along with complex (bidirectional) interactions accentuates the relevance of endothelial dysfunction $[29,30]$ in the pathogenesis of HFpEF.
Impact of Endothelial Dysfunction on Cardiac Fibroblasts

\section{Cardiac Inflammation Triggers Cardiac Fibroblasts}

Endothelial dysfunction/activation may contribute to cardiac fibrosis by different means. Murdoch et al. [31] recently demonstrated the role of endothelial NOX2 in the induction of cardiac inflammation and fibrosis in angiotensin II (Ang II) mice. Endothelium NOX2 transgenic mice had more vascular cell adhesion molecule (VCAM)-1 positive blood vessels and inflammatory cells after Ang II treatment than wild-type mice. In vitro co-culture of endothelial cells overexpressing NOX2 with cardiac fibroblasts provided further evidence that the induction of endothelial oxidative stress on its own is not responsible for the induction of cardiac fibrosis rather the stimulated inflammatory process. Since NO is capable of reducing oxidative stress on the one hand and decreasing the expression of monocyte chemoattractant protein-1 [9], VCAM-1 [10], and subsequent adhesion of immune cells [11] on the other hand, impaired NO bioavailability facilitates the engraftment of immune cells. Subsequently, engrafted immune cells may trigger cardiac fibrosis via inducing the differentiation of fibroblasts into myofibroblasts, which is reviewed in detail elsewhere [32••]. The importance of inflammation in the induction of cardiac fibrosis and heart failure, and particularly the role of the spleen as monocyte reservoir, has recently been broadly demonstrated [33-35]. In brief, we demonstrated that upon coculture with fibroblasts, splenocytes isolated from mice with inflammatory cardiomyopathy induce a higher collagen production in fibroblasts compared to splenocytes from control mice [34]. Ismahil et al. [33] showed that myocardial infarction is associated with cardiac inflammation and concomitant depletion of splenic monocytes. Furthermore, splenectomy experiments and adoptive transfer of splenocytes from mice with heart failure in naive recipients illustrated that splenocytes retain memory upon adoptive transfer and subsequently promote immune-mediated fibrosis in the failing heart. Later, van der Laan et al. [35] confirmed this unique spatiotemporal pattern of monocyte accumulation in the myocardium following myocardial infarction, which coincides with a marked depletion of monocytes from the spleen, in humans via analysis of human post-mortem specimens of myocardium, spleen, and bone marrow. Similar to splenocytes from myocarditis mice [34], we demonstrated that co-culture of splenocytes from Ang II-induced HFpEF mice induced a $46 \%$ higher collagen content in fibroblasts compared to splenocytes from control mice. This suggests that also in the pathogenesis of HFpEF, the cardiosplenic axis may be relevant. With 
respect to HFpEF, Westermann et al. [36] provided clear evidence linking cardiac inflammation with TGF- $\beta 1$ induced collagen synthesis and diastolic dysfunction in human subjects [37]. The role of TGF- $\beta$ as a profibrotic factor and its importance in the induction of diastolic function follows from previous experiments whereby the use of an anti-TGF- $\beta$-neutralizing antibody prevented myocardial fibrosis and diastolic dysfunction in pressure-overloaded rats [38].

Besides immune cells, also fibrocytes, circulating monocyte-derived cells with tissue remodeling properties of fibroblasts [39], which are induced in cardiovascular disorders $[39,40]$, can adhere to the activated endothelium, engraft in the heart, and contribute to cardiac fibrosis.

\section{Impaired Nitric Oxide Bioavailability Triggers Cardiac Fibroblasts}

Secondly, endothelial dysfunction contributes to cardiac fibrosis via the reduced bioavailability of NO, known to exert direct anti-fibrotic effects involving the cyclic guanosine monophosphate (cGMP) pathway [41, 42]. Vettel et al. [42] recently demonstrated that the cGMP/cyclic adenosine monophosphate (cAMP)-hydrolyzing phosphodiesterase (PDE) 2, which is upregulated in human failing hearts [43], leads to a decrease in cAMP levels in cardiac fibroblasts and accelerates the conversion of fibroblasts to myofibroblasts. Exogenous activation of cGMP via atrial natriuretic peptide (ANP) or NO was able to bypass the PDE2-mediated degradation of cAMP and reversed the differentiation of fibroblasts into myofibroblasts. These data emphasize the benefit of cGMP-elevating agents in the treatment of HFpEF which, besides their well-recognized merit for cardiomyocytes, can also be beneficial for cardiac fibroblasts. PDE5A inhibition with sildenafil counteracted cardiac hypertrophy and adverse remodeling in Ang II mice, an effect which was also associated with less cardiac inflammation [44]. These antiinflammatory effects of sildenafil, corroborated by Rizzo et al. [45], consequently indirectly further support the role of cardiac inflammation in cardiac remodeling and LV performance. Furthermore, these findings confirm that besides cardiomyocytes, also fibroblasts [46] and endothelial cells [45] are target cells of sildenafil. Despite abovementioned promising experimental findings [44], sildenafil failed to raise cGMP levels and to ameliorate LV diastolic function in HFpEF patients [47].

\section{Endothelial-to-Mesenchymal Transition}

Thirdly, NO deprivation [20, 48] leads to endothelial-tomesenchymal transition (EndMT), a process whereby endothelial cells convert to a mesenchymal cell type, which can give rise to fibroblasts. EndMT is induced by inflammatory factors (TGF- $\beta$ [49] and TNF- $\alpha$ [50]), oxidized LDL [51], and age [50]. In contrast, we could demonstrate that HDL decrease EndMT. Zeisberg et al. (2007) [49] provided first evidence that EndMT contributes to cardiac fibrosis during chronic pressure overload. Recently, endothelial-specific expression of endothelin-1 [52] and of NOX 2 [31] has been shown to induce EndMT in experimental models of diabetes mellitus and Ang II-induced cardiac hypertrophy and fibrosis, respectively. Intriguingly, Ang II-induced endothelial NOX2 activation was associated with isolated diastolic dysfunction in the absence of systolic dysfunction.

In summary, these findings suggest that endothelial dysfunction/activation and downstream pathways including not only inflammation but also far less appreciated EndMT, are important mechanisms contributing to cardiac fibrosis and the development of HFpEF [31]. Cardiac fibroblasts in turn can further trigger cardiac inflammation [53] in a multimodal manner which is reviewed in detail elsewhere [32••]. This complex interaction among endothelial cells, inflammatory cells, and cardiac fibroblasts might explain why unidirected strategies [54] counteracting inflammation or fibrosis have failed so far to block the fibrotic process and indicates the need for new strategies with more broaden immunomodulatory effects $[32 \bullet \cdot]$. Furthermore, it stresses the need of diagnosing and treating $\mathrm{HFpEF}$ at an early stage of its pathogenesis, where the vicious circle of inflammation and fibrosis, leading to chronic inflammation, might still be abrogated.

\section{Impact of Endothelial Dysfunction on Cardiomyocytes}

The endocardial endothelial cells and the endothelial cells of intramyocardial capillaries regulate the contractile state of cardiomyocytes via autocrine and paracrine signaling involving NO and endothelin-1. Furthermore, co-culture of cardiac endothelial cells with cardiomyocytes protects those cardiomyocytes from hydrogen peroxide-induced apoptosis through neuregulin-erbB4 signaling [55]. This indicates that cardiac endothelial cells exert direct anti-apoptotic effects in addition to their role in oxygen supply, which is needed for cardiomyocyte survival. Cardiac endothelial cells also guide cardiomyocyte organization and promote physiological coupling of cardiomyocytes and their synchronized contraction via influencing the expression of the principal gap junction protein connexin 43 [56]. On the other hand, upon inflammation [57] and mechanical load [58,59], endothelial cells may contribute to cardiomyocyte hypertrophy via the release of endothelin-1. Cardiomyocytes in turn can affect the coronary vasculature via multiple paracrine signals including endothelin-1 and fibroblast growth factor 2 [60, 61]. In addition, cardiomyocytes affect long-term growth and development of coronary arterial, venous, and lymphatic trees in which the paracrine release of VEGF-A by cardiomyocytes is of particular importance [60,62]. This cardiomyocyte- 
vascular crosstalk plays a critical role in the vascular adaptation, which takes place during myocardial hypertrophy. An imbalance between vasculature and cardiomyocyte growth may lead to progressive cardiac dysfunction and heart failure [60]. In brief, the above findings indicate the delicate balance between endothelial cells and cardiomyocytes and imply that changes due to endothelial dysfunction or deterioration may contribute to cardiomyocyte hypertrophy and HFpEF.

Particularly, the contribution of cardiomyocyte stiffness in diastolic LV stiffness and HFpEF is established. Besides intracellular calcium dysregulation, involving dysfunction of the sarcoplasmic reticular adenosine triphosphate (ATP)-driven pump (SERCA), phospholamban (PLB), and/or ryanodine receptor (RYR) 2, cardiomyocyte stiffness is mainly regulated by the giant sarcomeric protein titin [63]. Titin spans the sarcomere from the $\mathrm{Z}$ disk to the $\mathrm{M}$ line and functions as a molecular spring supporting early diastolic recoil and late diastolic distensibility of cardiomyocytes [64]. Evidence that solely titin stiffness is sufficient to induce diastolic dysfunction and $\mathrm{HFpEF}$, independent of extensive collagen deposition, follows from recent experimental studies. Chung et al. generated mice with a deletion of nine immunoglobulin-like domains from the proximal tandem immunoglobulin segment of the titin spring region, which resulted in overall titin stiffness [6•]. These knockout mice developed HFpEF despite unaltered myocardial collagen content. In addition, Hamdani et al. [65] recently demonstrated the importance of titin stiffness in the induction of diastolic dysfunction independently of cardiac fibrosis in a rat model of the metabolic syndrome. Besides shortage of titin length by experimental deletion of immunoglobulin-like domains [6 ${ }^{\bullet}$ or by oxidative stressinduced formation of disulfide bridges within the titin molecule [66], titin stiffness is also attributed to isoform shifts or posttranscriptional modifications like phosphorylation or oxidation [64]. Protein kinase $C \alpha$ phosphorylates titin at its proline, glutamate, valine, and lysine (PEVK) titin region and raises the stiffness of cardiomyoctes from normal myocardium [67], but not from cardiomyocytes isolated from an animal model of HFpEF [68]. On the other hand, the protein kinase extracellular signal-regulated kinase $2[69]$ and $\mathrm{Ca}^{2+} /$ calmodulin-dependent protein kinase II [70] phosphorylate titin and lower cardiomyocyte stiffness, though their pathophysiological relevance for HFpEF needs to be further clarified. In HFpEF patients, titin at the stiff N2B isoform is hypophosphorylated [71]. Protein kinases A [72] and G [73, 74•.] phosphorylate titin at its N2B segment, and lower cardiomyocyte stiffness, which implies that $\beta$ adrenergic stimulation, $\mathrm{NO}$, and natriuretic peptides may decrease cardiomyocyte stiffness. With respect to $\mathrm{Ca}^{2+} /$ calmodulin-dependent protein kinase II and its involvement in $\mathrm{NO}$ synthesis as a result of $\mathrm{Ca}^{2+}$-dependent activation of eNOS [75], it is tempting to speculate that $\mathrm{Ca}^{2+} /$ calmodulin-dependent protein kinase II may further contribute to the relaxation of titin via triggering NO release. Due to the potential risk of arrhythmic death, the induction of protein kinase $A$ via $B$ adrenergic stimulation is excluded as therapeutical option. Blocking the breakdown of the downstream NO target cGMP via sildenafil has not been successful in HFpEF patients despite promising experimental studies (see infra). In contrast, favorable effects in improving diastolic dysfunction in patients with HFpEF have recently been found by the use of the neprilysin inhibitor (LCZ696), which inhibits the breakdown of natriuretic peptides. Neprilysin activity is induced in obese patients [76]. The finding that IL- $1 ß$ induces neprilysin activity [77] may explain the increased neprilysin and B-type natriuretic peptide (BNP) paradox in obese HFpEF patients, which is associated with increased inflammation [78].

In summary, the importance of NO bioavailability and oxidative stress in titin relaxation (of the N2B segment) [74.•] as well as the role of inflammation in the induction of neprilysin activity [77] and subsequent impaired natriuretic signaling further corroborates the impact of endothelial dysfunction on cardiomyocyte stiffness.

Impact of Endothelial Dysfunction on Cardiac Neurohumoral Regulation

As it has already been broadly outlined before, HFpEF comorbidities provoke a systemic inflammatory state, which severely affects the coronary microvascular endothelium [36, 79]. The sum outcome of microvascular deterioration is neuronal dysfunction and death as well as the reduction of neurovascular perfusion, functional impairment, and cellular apoptosis [80]. Inflammatory and hyperglycemia-induced oxidative stress in vascular endothelial cells correlates with cardiac autonomic nerve dysfunction [81]. eNOS uncoupling leading to nitrosative stress and formation of peroxynitrite induces damage of neuronal axons, which are particularly prone to oxidative and nitrosative stress due to their high mitochondrial content [82]. This leads to a prominent abnormality in the levels of cardioactive neuropeptides including neuropeptide $\mathrm{Y}$, substance $\mathrm{P}$ [83], calcitonin-generated peptide, natriuretic peptides (ANP, BNP, and C-type natriuretic peptide) [84] as well as the respective neuropeptide effectors [85], contributing to impaired endothelium-dependent vasodilation in HFpEF [86]. This limits the delivery and extraction of tissue oxygen, which subsequently results in nerve hypoxia and impaired nerve function [80]. The damage of the nerve fibers innervating the heart and blood vessels, common for cardiovascular autonomic neuropathy, results in abnormalities in heart rate control and vascular dynamics. Besides their role as vasodilatory proteins, the natriuretic peptides ANP and BNP exert anti-fibrotic [87] and anti-hypertrophic effects [88] and influence titin stiffness. Under metabolic conditions, 
BNP degradation seems to be paradoxically increased [89], contributing to cardiac fibrosis and cardiomyocyte stiffness.

Cardiovascular autonomic neuropathy in diabetes mellitus is associated with LV diastolic and systolic dysfunction, LV hypertrophy, and higher LV mass index [90]. Findings from studies of the autonomic nervous system indicate that it can modulate inflammatory reactions [91]. These findings suggest that dysregulation of the cardiac neurohumoral system not only directly modulates vascular dynamics and heart function but also activates cardiac inflammation and subsequent remodeling.

\section{Conclusions and Perspectives}

HFpEF is a complex disorder caused by multifactorial stresses secondary to comorbidities. This review highlights the importance of endothelial dysfunction or deterioration at the early onset of the pathogenesis of HFpEF and stresses the subsequent complexity of cardiac remodeling due to bidirectional interactions between endothelial cells, cardiomyocytes, fibroblasts, and cardiac neurohumoral activation. It further strengthens the need for an early diagnosis of endothelial dysfunction [92 •*] enabling treatment before the development of HFpEF-specific cardiac remodeling. So far, only the prevention of HFpEF through treatment of risk factors has been shown to be effective [93]. It will be the challenge of finding new multidirectional strategies to abrogate endothelial dysfunction and subsequent cardiac remodeling. Given the importance of inflammation in the induction of endothelial dysfunction and cardiac remodeling via the cardiosplenic axis [33, 34], intravenous application of mesenchymal stromal cells, and the recently identified cardiac-derived adherent proliferating cells (CardAPs) [94] having immunomodulatory [34, 95-97], endothelium-protective [98], and anti-fibrotic [34, 99, 100] features might be attractive tools to counteract the inflammatory and cardiac remodeling process.

Acknowledgments This study is supported by the DZHK to SVL and CT and by the European 7th Framework Consortia MEDIA and REDDSTAR to CT. We would like to thank Dr. Kapka Miteva for her contribution to the paragraph focused on cardiac neurohumoral activation and for the artwork.

\section{Compliance with Ethics Guidelines}

Conflict of Interest Carsten Tschöpe and Sophie Van Linthout declare that they have no conflict of interest.

Human and Animal Rights and Informed Consent This review includes original data from a study, performed by the authors, that was conducted with animals. The study was approved by the local ethics committee (Landesamt für Gesundheit und Soziales, Tierschutz, Berlin).
Open Access This article is distributed under the terms of the Creative Commons Attribution License which permits any use, distribution, and reproduction in any medium, provided the original author(s) and the source are credited.

\section{References}

Papers of particular interest, published recently, have been highlighted as:

- Of importance

-. Of major importance

1. Owan TE, Hodge DO, Herges RM, et al. Trends in prevalence and outcome of heart failure with preserved ejection fraction. N Engl J Med. 2006;355:251-9.

2. Borlaug BA, Paulus WJ. Heart failure with preserved ejection fraction: pathophysiology, diagnosis, and treatment. Eur Heart J. 2011;32:670-9.

3. King H, Aubert RE, Herman WH. Global burden of diabetes, 1995-2025: prevalence, numerical estimates, and projections. Diabetes Care. 1998;21:1414-31.

4. Flegal KM, Carroll MD, Kit BK, Ogden CL. Prevalence of obesity and trends in the distribution of body mass index among US adults, 1999-2010. JAMA. 2012;307:491-7.

5. Hamdani N, Paulus WJ. Myocardial titin and collagen in cardiac diastolic dysfunction: partners in crime. Circulation. 2013;128:58 .

6. Chung CS, Hutchinson KR, Methawasin M, et al. Shortening of the elastic tandem immunoglobulin segment of titin leads to diastolic dysfunction. Circulation. 2013;128:19-28. This article provides first evidence that titin stiffness, in the absence of any involvement of the extracellular matrix, is sufficient to induce HFpEF.

7.• Paulus WJ, Tschope C. A novel paradigm for heart failure with preserved ejection fraction: comorbidities drive myocardial dysfunction and remodeling through coronary microvascular endothelial inflammation. J Am Coll Cardiol. 2013;62:263-71. This review postulates a new paradigm for the development of $H F p E F$, which identifies a systemic proinflammatory state induced by comorbidities as the origin of microvascular endothelial cell inflammation and subsequent concentric cardiac remodeling and dysfunction.

8. Brutsaert DL. Cardiac endothelial-myocardial signaling: its role in cardiac growth, contractile performance, and rhythmicity. Physiol Rev. 2003;83:59-115.

9. Zeiher AM, Fisslthaler B, Schray-Utz B, Busse R. Nitric oxide modulates the expression of monocyte chemoattractant protein 1 in cultured human endothelial cells. Circ Res. 1995;76:980-6.

10. Khan BV, Harrison DG, Olbrych MT, et al. Nitric oxide regulates vascular cell adhesion molecule 1 gene expression and redoxsensitive transcriptional events in human vascular endothelial cells. Proc Natl Acad Sci U S A. 1996;93:9114-9.

11. Niu XF, Smith CW, Kubes P. Intracellular oxidative stress induced by nitric oxide synthesis inhibition increases endothelial cell adhesion to neutrophils. Circ Res. 1994;74:1133-40.

12. Riad A, Westermann D, Van Linthout S, et al. Enhancement of endothelial nitric oxide synthase production reverses vascular dysfunction and inflammation in the hindlimbs of a rat model of diabetes. Diabetologia. 2008;51:2325-32.

13. Dimmeler S, Haendeler J, Nehls M, Zeiher AM. Suppression of apoptosis by nitric oxide via inhibition of interleukin-1beta- 
converting enzyme (ICE)-like and cysteine protease protein (CPP)-32-like proteases. J Exp Med. 1997;185:601-7.

14. Emanueli C, Van Linthout S, Salis MB, et al. Nitric oxidereleasing aspirin derivative, NCX 4016, promotes reparative angiogenesis and prevents apoptosis and oxidative stress in a mouse model of peripheral ischemia. Arterioscler Thromb Vasc Biol. 2004;24:2082-7.

15. Brunner H, Cockcroft JR, Deanfield J, et al. Endothelial function and dysfunction. Part II: association with cardiovascular risk factors and diseases. A statement by the Working Group on Endothelins and Endothelial Factors of the European Society of Hypertension. J Hypertens. 2005;23:233-46.

16. Goodwin BL, Pendleton LC, Levy MM, et al. Tumor necrosis factor-alpha reduces argininosuccinate synthase expression and nitric oxide production in aortic endothelial cells. Am J Physiol Heart Circ Physiol. 2007;293:H1115-21.

17. Gao X, Xu X, Belmadani S, et al. TNF-alpha contributes to endothelial dysfunction by upregulating arginase in ischemia/ reperfusion injury. Arterioscler Thromb Vasc Biol. 2007;27: 1269-75.

18. Spillmann F, Van Linthout S, Miteva K, et al. LXR agonism improves TNF-alpha-induced endothelial dysfunction in the absence of its cholesterol-modulating effects. Atherosclerosis. 2014;232:1-9.

19. Van Linthout S, Spillmann F, Lorenz M, et al. Vascular-protective effects of high-density lipoprotein include the downregulation of the angiotensin II type 1 receptor. Hypertension. 2009;53:682-7.

20. Almudever P, Milara J, De Diego A, et al. Role of tetrahydrobiopterin in pulmonary vascular remodelling associated with pulmonary fibrosis. Thorax. 2013;68:938-48.

21. Cai S, Khoo J, Mussa S, et al. Endothelial nitric oxide synthase dysfunction in diabetic mice: importance of tetrahydrobiopterin in eNOS dimerisation. Diabetologia. 2005;48:1933-40.

22. Gao X, Zhang H, Belmadani S, et al. Role of TNF-alphainduced reactive oxygen species in endothelial dysfunction during reperfusion injury. Am J Physiol Heart Circ Physiol. 2008;295:H2242-9.

23. $\mathrm{Xu} \mathrm{J,} \mathrm{Zou} \mathrm{MH.} \mathrm{Molecular} \mathrm{insights} \mathrm{and} \mathrm{therapeutic} \mathrm{targets} \mathrm{for}$ diabetic endothelial dysfunction. Circulation. 2009;120:1266-86.

24. Hsieh PC, Davis ME, Lisowski LK, Lee RT. Endothelialcardiomyocyte interactions in cardiac development and repair. Annu Rev Physiol. 2006;68:51-66.

25. Shah AM. Paracrine modulation of heart cell function by endothelial cells. Cardiovasc Res. 1996;31:847-67.

26. Zhang M, Shah AM. ROS signalling between endothelial cells and cardiac cells. Cardiovasc Res. 2014;102:249-57.

27. Brutsaert DL, Meulemans AL, Sipido KR, Sys SU. Effects of damaging the endocardial surface on the mechanical performance of isolated cardiac muscle. Circ Res. 1988;62:358-66.

28. Paulus WJ, Vantrimpont PJ, Shah AM. Paracrine coronary endothelial control of left ventricular function in humans. Circulation. 1995;92:2119-26.

29. Tschope C, Bock CT, Kasner M, et al. High prevalence of cardiac parvovirus B19 infection in patients with isolated left ventricular diastolic dysfunction. Circulation. 2005;111:879-86.

30. Lam CS, Brutsaert DL. Endothelial dysfunction: a pathophysiologic factor in heart failure with preserved ejection fraction. J Am Coll Cardiol. 2012;60:1787-9.

31. Murdoch CE, Chaubey S, Zeng L, et al. Endothelial NADPH oxidase-2 promotes interstitial cardiac fibrosis and diastolic dysfunction through pro-inflammatory effects and endothelialmesenchymal transition. J Am Coll Cardiol. 2014;63:2734-41.

32.• Van Linthout S, Miteva K, Tschope C. Crosstalk between fibroblasts and inflammatory cells. Cardiovasc Res. 2014;102:258-69. This review gives an extensive overview about how inflammatory cells contribute to (cardiac) fibroblast activation and how in turn fibroblasts contribute to the inflammatory process leading to chronic inflammation.

33. Ismahil MA, Hamid T, Bansal SS, et al. Remodeling of the mononuclear phagocyte network underlies chronic inflammation and disease progression in heart failure: critical importance of the cardiosplenic axis. Circ Res. 2014;114:266-82.

34. Savvatis K, van Linthout S, Miteva K, et al. Mesenchymal stromal cells but not cardiac fibroblasts exert beneficial systemic immunomodulatory effects in experimental myocarditis. PLoS One. 2012;7:e41047.

35. van der Laan AM, Ter Horst EN, Delewi R, et al. Monocyte subset accumulation in the human heart following acute myocardial infarction and the role of the spleen as monocyte reservoir. Eur Heart J. 2014;35:376-85.

36. Westermann D, Lindner D, Kasner M, et al. Cardiac inflammation contributes to changes in the extracellular matrix in patients with heart failure and normal ejection fraction. Circ Heart Fail. 2011;4: 44-52.

37. Kapur NK. Transforming growth factor-beta: governing the transition from inflammation to fibrosis in heart failure with preserved left ventricular function. Circ Heart Fail. 2011;4:5-7.

38. Kuwahara F, Kai H, Tokuda K, et al. Transforming growth factorbeta function blocking prevents myocardial fibrosis and diastolic dysfunction in pressure-overloaded rats. Circulation. 2002;106: $130-5$.

39. Reilkoff RA, Bucala R, Herzog EL. Fibrocytes: emerging effector cells in chronic inflammation. Nat Rev Immunol. 2011;11:42735 .

40. Williams SM, Golden-Mason L, Ferguson BS, et al. Class I HDACs regulate angiotensin II-dependent cardiac fibrosis via fibroblasts and circulating fibrocytes. J Mol Cell Cardiol. 2014;67:112-25.

41. Li P, Wang D, Lucas J, et al. Atrial natriuretic peptide inhibits transforming growth factor beta-induced Smad signaling and myofibroblast transformation in mouse cardiac fibroblasts. Circ Res. 2008;102:185-92.

42. Vettel C, Lammle S, Ewens S, et al. PDE2-mediated cAMP hydrolysis accelerates cardiac fibroblast to myofibroblast conversion and is antagonized by exogenous activation of cGMP signaling pathways. Am J Physiol Heart Circ Physiol. 2014;306: H1246-52.

43. Mehel H, Emons J, Vettel C, et al. Phosphodiesterase-2 is upregulated in human failing hearts and blunts beta-adrenergic responses in cardiomyocytes. J Am Coll Cardiol. 2013;62:1596606.

44. Westermann D, Becher PM, Lindner D, et al. Selective PDE5A inhibition with sildenafil rescues left ventricular dysfunction, inflammatory immune response and cardiac remodeling in angiotensin IIinduced heart failure in vivo. Basic Res Cardiol. 2012;107:308.

45. Rizzo NO, Maloney E, Pham M, et al. Reduced NO-cGMP signaling contributes to vascular inflammation and insulin resistance induced by high-fat feeding. Arterioscler Thromb Vasc Biol. 2010;30:758-65.

46. Dunkern TR, Feurstein D, Rossi GA, et al. Inhibition of TGF-beta induced lung fibroblast to myofibroblast conversion by phosphodiesterase inhibiting drugs and activators of soluble guanylyl cyclase. Eur J Pharmacol. 2007;572:12-22.

47. Redfield MM, Chen HH, Borlaug BA, et al. Effect of phosphodiesterase- 5 inhibition on exercise capacity and clinical status in heart failure with preserved ejection fraction: a randomized clinical trial. JAMA. 2013;309:1268-77.

48. O'Riordan E, Mendelev N, Patschan S, et al. Chronic NOS inhibition actuates endothelial-mesenchymal transformation. Am J Physiol Heart Circ Physiol. 2007;292:H285-94.

49. Zeisberg EM, Tarnavski O, Zeisberg M, et al. Endothelial-tomesenchymal transition contributes to cardiac fibrosis. Nat Med. 2007;13:952-61. 
50. Fleenor BS, Marshall KD, Rippe C, Seals DR. Replicative aging induces endothelial to mesenchymal transition in human aortic endothelial cells: potential role of inflammation. J Vasc Res. 2012;49:59-64.

51. Kim M, Choi SH, Jin YB, et al. The effect of oxidized low-density lipoprotein (ox-LDL) on radiation-induced endothelial-tomesenchymal transition. Int J Radiat Biol. 2013;89:356-63.

52. Widyantoro B, Emoto N, Nakayama K, et al. Endothelial cellderived endothelin-1 promotes cardiac fibrosis in diabetic hearts through stimulation of endothelial-to-mesenchymal transition. Circulation. 2010;121:2407-18.

53. Lindner D, Zietsch C, Tank J, et al. Cardiac fibroblasts support cardiac inflammation in heart failure. Basic Res Cardiol. 2014;109:428.

54. Javed Q, Murtaza I. Therapeutic potential of tumour necrosis factor-alpha antagonists in patients with chronic heart failure. Heart Lung Circ. 2013;22:323-7.

55. Kuramochi Y, Cote GM, Guo X, et al. Cardiac endothelial cells regulate reactive oxygen species-induced cardiomyocyte apoptosis through neuregulin-1beta/erbB4 signaling. J Biol Chem. 2004;279:51141-7.

56. Narmoneva DA, Vukmirovic R, Davis ME, et al. Endothelial cells promote cardiac myocyte survival and spatial reorganization: implications for cardiac regeneration. Circulation. 2004;110:962-8.

57. Scalera F. Intracellular glutathione and lipid peroxide availability and the secretion of vasoactive substances by human umbilical vein endothelial cells after incubation with TNF-alpha. Eur J Clin Investig. 2003;33:176-82.

58. van Wamel AJ, Ruwhof C, van der Valk-Kokshoorn LJ, et al. Stretch-induced paracrine hypertrophic stimuli increase TGFbetal expression in cardiomyocytes. Mol Cell Biochem. 2002;236:147-53.

59. van Wamel AJ, Ruwhof C, van der Valk-Kokshoom LE, et al. The role of angiotensin II, endothelin-1 and transforming growth factor-beta as autocrine/paracrine mediators of stretchinduced cardiomyocyte hypertrophy. Mol Cell Biochem. 2001;218:113-24.

60. Tirziu D, Giordano FJ, Simons M. Cell communications in the heart. Circulation. 2010;122:928-37.

61. Zhou M, Sutliff RL, Paul RJ, et al. Fibroblast growth factor 2 control of vascular tone. Nat Med. 1998;4:201-7.

62. Giordano FJ, Gerber HP, Williams SP, et al. A cardiac myocyte vascular endothelial growth factor paracrine pathway is required to maintain cardiac function. Proc Natl Acad Sci U S A. 2001;98: 5780-5.

63. Linke WA. Sense and stretchability: the role of titin and titinassociated proteins in myocardial stress-sensing and mechanical dysfunction. Cardiovasc Res. 2008;77:637-48.

64. LeWinter MM, Granzier H. Cardiac titin: a multifunctional giant. Circulation. 2010;121:2137-45.

65. Hamdani N, Franssen C, Lourenco A, et al. Myocardial titin hypophosphorylation importantly contributes to heart failure with preserved ejection fraction in a rat metabolic risk model. Circ Heart Fail. 2013;6:1239-49.

66. Grutzner A, Garcia-Manyes S, Kotter S, et al. Modulation of titinbased stiffness by disulfide bonding in the cardiac titin N2-B unique sequence. Biophys J. 2009;97:825-34.

67. Hidalgo C, Hudson B, Bogomolovas J, et al. PKC phosphorylation of titin's PEVK element: a novel and conserved pathway for modulating myocardial stiffness. Circ Res. 2009;105:631-8. $17 p$ following 638 .

68. Hamdani N, Bishu KG, von Frieling-Salewsky M, et al. Deranged myofilament phosphorylation and function in experimental heart failure with preserved ejection fraction. Cardiovasc Res. 2013;97: 464-71.
69. Raskin A, Lange S, Banares K, et al. A novel mechanism involving four-and-a-half LIM domain protein-1 and extracellular signal-regulated kinase-2 regulates titin phosphorylation and mechanics. J Biol Chem. 2012;287:29273-84.

70. Hamdani N, Krysiak J, Kreusser MM, et al. Crucial role for Ca2(+ )/calmodulin-dependent protein kinase-II in regulating diastolic stress of normal and failing hearts via titin phosphorylation. Circ Res. 2013;112:664-74.

71. Borbely A, Falcao-Pires I, van Heerebeek L, et al. Hypophosphorylation of the Stiff N2B titin isoform raises cardiomyocyte resting tension in failing human myocardium. Circ Res. 2009;104:780-6.

72. Fukuda N, Wu Y, Nair P, Granzier HL. Phosphorylation of titin modulates passive stiffness of cardiac muscle in a titin isoformdependent manner. J Gen Physiol. 2005;125:257-71.

73. Kruger M, Kotter S, Grutzner A, et al. Protein kinase G modulates human myocardial passive stiffness by phosphorylation of the titin springs. Circ Res. 2009;104:87-94.

74.•• van Heerebeek L, Hamdani N, Falcao-Pires I, et al. Low myocardial protein kinase $\mathrm{G}$ activity in heart failure with preserved ejection fraction. Circulation. 2012;126:830-9. This article demonstrated reduced myocardial cGMP concentration and protein kinase $G$ activity in HFpEF compared to HFREF patients.

75. Schneider JC, El Kebir D, Chereau C, et al. Involvement of Ca2+/ calmodulin-dependent protein kinase II in endothelial NO production and endothelium-dependent relaxation. Am J Physiol Heart Circ Physiol. 2003;284:H2311-9.

76. Das SR, Drazner MH, Dries DL, et al. Impact of body mass and body composition on circulating levels of natriuretic peptides: results from the Dallas Heart Study. Circulation. $2005 ; 112: 2163-8$.

77. Kondepudi A, Johnson A. Cytokines increase neutral endopeptidase activity in lung fibroblasts. Am J Respir Cell Mol Biol. 1993;8:43-9.

78. Carbone S, Shah KB, Van Tassell BW, et al. Obesity and diastolic heart failure: is inflammation the link? Transl Med. 2013;3:e124.

79. van Heerebeek L, Hamdani N, Handoko ML, et al. Diastolic stiffness of the failing diabetic heart: importance of fibrosis, advanced glycation end products, and myocyte resting tension. Circulation. 2008;117:43-51.

80. Ramasamy R, Vannucci SJ, Yan SS, et al. Advanced glycation end products and RAGE: a common thread in aging, diabetes, neurodegeneration, and inflammation. Glycobiology. 2005;15:16R28R.

81. Ziegler D, Buchholz S, Sohr C, et al. Oxidative stress predicts progression of peripheral and cardiac autonomic nerve dysfunction over 6 years in diabetic patients. Acta Diabetol. 2014. doi:10. 1007/s00592-014-0601-3.

82. Leinninger GM, Edwards JL, Lipshaw MJ, Feldman EL. Mechanisms of disease: mitochondria as new therapeutic targets in diabetic neuropathy. Nat Clin Pract Neurol. 2006;2:620-8.

83. Kunt T, Forst T, Schmidt S, et al. Serum levels of substance $P$ are decreased in patients with type 1 diabetes. Exp Clin Endocrinol Diabetes. 2000;108:164-7.

84. McKenna K, Smith D, Tormey W, Thompson CJ. Acute hyperglycaemia causes elevation in plasma atrial natriuretic peptide concentrations in type 1 diabetes mellitus. Diabet Med. 2000;17:512-7.

85. Chottova Dvorakova M, Wiegand S, Pesta M, et al. Expression of neuropeptide $\mathrm{Y}$ and its receptors $\mathrm{Y} 1$ and $\mathrm{Y} 2$ in the rat heart and its supplying autonomic and spinal sensory ganglia in experimentally induced diabetes. Neuroscience. 2008;151:1016-28.

86. Borlaug BA, Olson TP, Lam CS, et al. Global cardiovascular reserve dysfunction in heart failure with preserved ejection fraction. J Am Coll Cardiol. 2010;56:845-54. 
87. Kapoun AM, Liang F, O'Young G, et al. B-type natriuretic peptide exerts broad functional opposition to transforming growth factorbeta in primary human cardiac fibroblasts: fibrosis, myofibroblast conversion, proliferation, and inflammation. Circ Res. 2004;94: 453-61.

88. Struthers AD. Ten years of natriuretic peptide research: a new dawn for their diagnostic and therapeutic use? BMJ. 1994;308:1615-9.

89. Mehra MR, Uber PA, Park MH, et al. Obesity and suppressed Btype natriuretic peptide levels in heart failure. J Am Coll Cardiol. 2004;43:1590-5.

90. Taskiran M, Rasmussen V, Rasmussen B, et al. Left ventricular dysfunction in normotensive type 1 diabetic patients: the impact of autonomic neuropathy. Diabet Med. 2004;21:524-30.

91. Tracey KJ. The inflammatory reflex. Nature. 2002;420:853-9.

92.• Akiyama E, Sugiyama S, Matsuzawa Y, et al. Incremental prognostic significance of peripheral endothelial dysfunction in patients with heart failure with normal left ventricular ejection fraction. J Am Coll Cardiol. 2012;60:1778-86. This article outlines the prognostic significance of peripheral endothelial dysfunction in patients with $H F p E F$.

93. Schocken DD, Benjamin EJ, Fonarow GC, et al. Prevention of heart failure: a scientific statement from the American Heart Association Councils on Epidemiology and Prevention, Clinical Cardiology, Cardiovascular Nursing, and High Blood Pressure Research; Quality of Care and Outcomes Research Interdisciplinary Working Group; and Functional Genomics and
Translational Biology Interdisciplinary Working Group. Circulation. 2008;117:2544-65.

94. Haag M, Van Linthout S, Schroder SE, et al. Endomyocardial biopsy derived adherent proliferating cells - a potential cell source for cardiac tissue engineering. J Cell Biochem. 2010;109:564-75.

95. Ren G, Zhang L, Zhao X, et al. Mesenchymal stem cell-mediated immunosuppression occurs via concerted action of chemokines and nitric oxide. Cell Stem Cell. 2008;2:141-50.

96. Van Linthout S, Savvatis K, Miteva K, et al. Mesenchymal stem cells improve murine acute coxsackievirus B3-induced myocarditis. Eur Heart J. 2011;32:2168-78.

97. Haag M, Stolk M, Ringe J, et al. Immune attributes of cardiacderived adherent proliferating (CAP) cells in cardiac therapy. $\mathrm{J}$ Tissue Eng Regen Med. 2013;7:362-70.

98. Prather WR, Toren A, Meiron $\mathrm{M}$, et al. The role of placental-derived adherent stromal cell (PLX-PAD) in the treatment of critical limb ischemia. Cytotherapy. 2009;11: 427-34.

99. Mias C, Lairez O, Trouche E, et al. Mesenchymal stem cells promote matrix metalloproteinase secretion by cardiac fibroblasts and reduce cardiac ventricular fibrosis after myocardial infarction. Stem Cells. 2009;27:2734-43.

100. Miteva K, Haag M, Peng J, et al. Human cardiac-derived adherent proliferating cells reduce murine acute coxsackievirus B3-induced myocarditis. PLoS One. 2011;6:e28513. 